\title{
Integration of Real-Time and Off-Line Clinical Data in the MIMIC Database
}

\author{
GB Moody, RG Mark
}

\author{
Harvard-MIT Division of Health Sciences and Technology, Cambridge, MA, USA \\ Cardiology Division, Beth Israel Deaconess Medical Center, Boston, MA, USA
}

\begin{abstract}
The MIMIC (Multi-parameter Intelligent Monitoring for Intensive Care) Database is a collection of recorded physiologic signals and clinical data intended to support development and evaluation of automated decision support systems for the intensive care setting. Each patient record typically includes 24 to 48 hours of continuous real-time signals (e.g., ECGs, blood pressure waveforms, respiration) and derived measurements (e.g., heart rate, systolic BP), together with clinical data derived from the patient's medical record and from the hospital's clinical computing systems. The clinical data are encoded using structured HTML (readable using a standard web browser, or by a decision support system or other automated application). An important element of this encoding is a reconstruction of the medical record in the form of a time-line of events, which permits a detailed and repeatable simulation of the inputs that are potentially available to a real-time "intelligent" ICU monitor.
\end{abstract}

\section{Introduction}

"Intelligent" patient monitoring and decision support systems for the intensive care setting will begin to be developed during the next several years. The clinical problems presented in the intensive care unit (ICU) often require rapid assimilation of a large amount of information from a wide variety of sources. For example, given a sudden drop in blood pressure, a timely intervention is required, but the choice of appropriate action may depend on knowledge of the patient's cardiac rhythm, fluid balance, short- and long-term trends in vital signs, medications given recently, likelihood of hemorrhage or sepsis, and the patient's response to previous interventions, in addition to a good understanding of the patient's baseline status and major medical problems. Patient monitoring systems that can assist in the formidable tasks of gathering, organizing, and presenting this information, tracking and anticipating changes in patient status, formulating clinical hypotheses in real-time, and offering support for decision making, are becoming increasingly necessary as physicians and nurses face what has been called information overload in the ICU.

Progress towards the goal of comprehensive computer-readable medical records, considered together with the clinical and economic incentives for achieving this goal, suggest that on-line medical records may become a reality in many hospitals within the next few years. Future generations of patient monitoring systems will need to access these records in order to provide competent decision support, for the same reasons that physicians and nurses consult existing medical records. The MIMIC Database [1] is intended to support development of intelligent patient monitoring systems for the ICU. In anticipation of the eventual widespread availability of on-line medical records, we have chosen to design the MIMIC Database as if such records were available already.

The MIMIC Database contains 91 patient records obtained in the intensive care units (ICUs) of Boston's Beth Israel Hospital (now the Beth Israel Deaconess Medical Center). Of the records in the MIMIC Database, the most common major diagnoses are respiratory failure (20 cases), congestive heart failure with pulmonary edema (15 cases), and sepsis (11 cases). Many other medical problems requiring intensive care are also represented.

\section{Methods}

Typical MIMIC Database records (archived on CDROMs) occupy 200-500 megabytes each, nearly all of which is needed for storage of the real-time signals. The clinical data, by contrast, typically require much less than 1 megabyte per patient, and consist of admitting notes (containing a synopsis of the patient's condition at the time of admission to the hospital, updated to the time of the beginning of the recording, 
with relevant history, demographic data, physical examination, and system review), results of laboratory tests, medication logs, physicians' and nurses' notes, flow sheets (hourly reports compiled by the ICU staff, including vital signs and measurements of intake and output needed to assess fluid balance), and discharge summaries.

Clinical data are entered into the MIMIC Database from two sources. First, data from the hospital's online clinical information systems are downloaded in text form. About 60 laboratory report formats are recognized by software we designed for this task, which parses the text stream and generates structured HTML as illustrated at the top of figure 1. Notably, all data obtained in this way are time-stamped (i.e., the date and time at which each datum was obtained has been recorded). A small amount of manual editing is required to produce finished hypertext files; in a largerscale operation, it would be economically justifiable to automate these editing operations as well. Certain laboratory tests are performed repeatedly; the results of each such test are gathered into a single hypertext file, as illustrated in the lower portion of figure 1.

The second source of clinical data is the off-line medical record. As in most hospitals, the patients' (paper) medical records contain a great deal of information that is never entered into our hospital's on-line clinical information systems. (Examples of such information include physicians' and nurses' progress notes, and times and dosages of medications given.) Relevant information from the paper record must be identified. This step requires review of the entire record, which often documents multiple hospital admissions in addition to clinic visits; from this large body of material we select the portions of the patient's medical history that are necessary for understanding the patient's state upon admission. The selected information is then manually transcribed into a relational database. At this stage, extensive error-checking is performed, in order to reduce the incidence of transcription errors as well as to detect and resolve inconsistencies in the original record. To the extent possible, we attempt in this stage to assign time stamps to the data extracted in this way from the paper record; in some cases, however, there may be considerable ambiguity (e.g., "the patient was more alert this morning") in the assignment of time stamps and even in determining the chronologic order of data that were originally recorded on different pieces of paper. After the reconstructed record as entered into the relational database has been thoroughly checked, it is then extracted and rewritten in structured HTML form. Again, software designed for this purpose does much of the work, but considerable man-
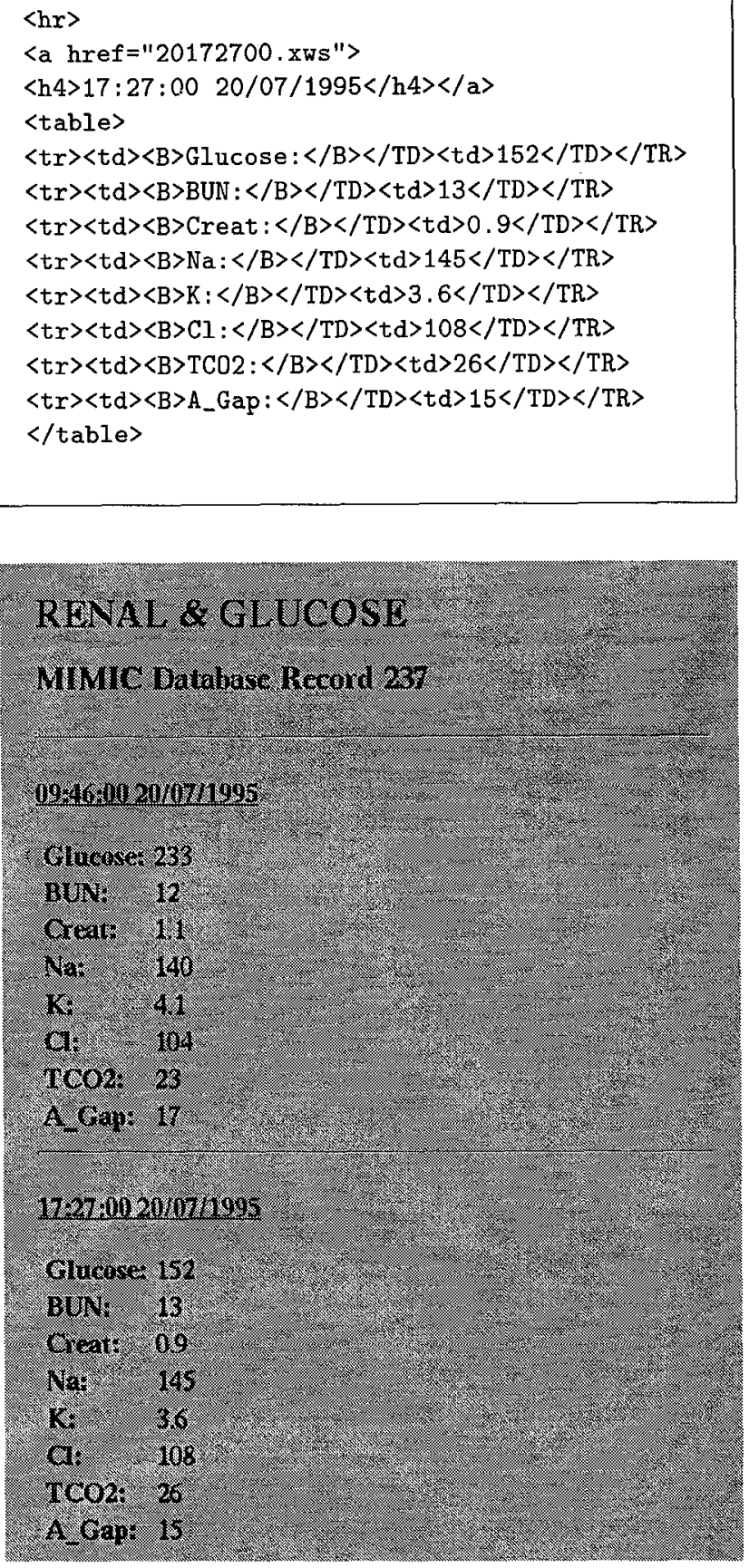

Figure 1: Top: Structured HTML fragment generated from a laboratory report. Note the hyperlink associated with the time stamp; this permits ready interactive access to the associated physiologic signals and measurements from a web browser working in cooperation with software such as WAVE (see figure 2). Bottom: A portion of the finished hypertext file, including the data shown at the top of the figure, viewed using a web browser. 


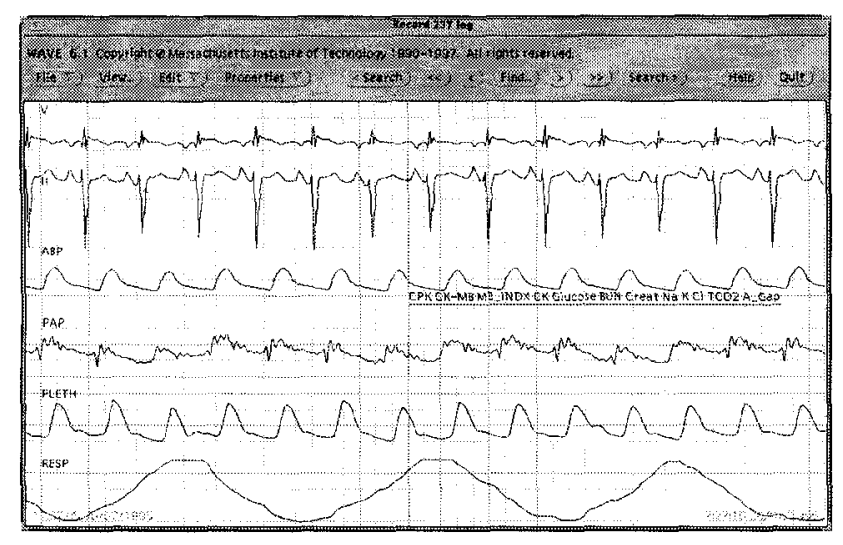

Figure 2: Waveform data at the time of the laboratory test shown in figure 1, as viewed using WAVE. The annotation at the center is linked to the HTML time-line file (see text); the variable names listed in the annotation's text specify which laboratory measurements were recorded at that time.

ual editing is required here. Regrettably, the relational database vendor's HTML output format is intended only for visual review; the structure of the data is very poorly represented in the output, necessitating extensive post-processing and manual correction. As for the data obtained from the on-line clinical information systems, it would be possible (though not justifiable on the scale of this project) to automate nearly all of the manual editing in this stage (because of known bugs in the relational database vendor's HTML output, however, it would still be necessary to perform manual correction to restore truncated data in some cases).

Once all of the clinical data have been incorporated into structured HTML files with time stamps, we reconstruct the medical record in the form of a timeline of events (also encoded as an HTML file). The HTML time-line permits a detailed and repeatable simulation of the inputs that are potentially available to a real-time "intelligent" ICU monitor. From the HTML time-line file, we prepare an annotation file to accompany the recorded signals; this annotation file can be displayed directly by software such as WAVE $[1,2]$ (see figure 2). The annotation file contains an annotation for each time stamp in the time line, with an attached hyperlink to the HTML time-line file. Recent versions of WAVE work cooperatively with a web browser. By selecting a hyperlinked annotation using WAVE, the web browser can be launched if necessary, and made to display the corresponding portion of the HTML time line file (see figure 3). Similarly, by selecting a timestamp hyperlink within the web browser, WAVE can be launched if necessary, and made to display the cor-

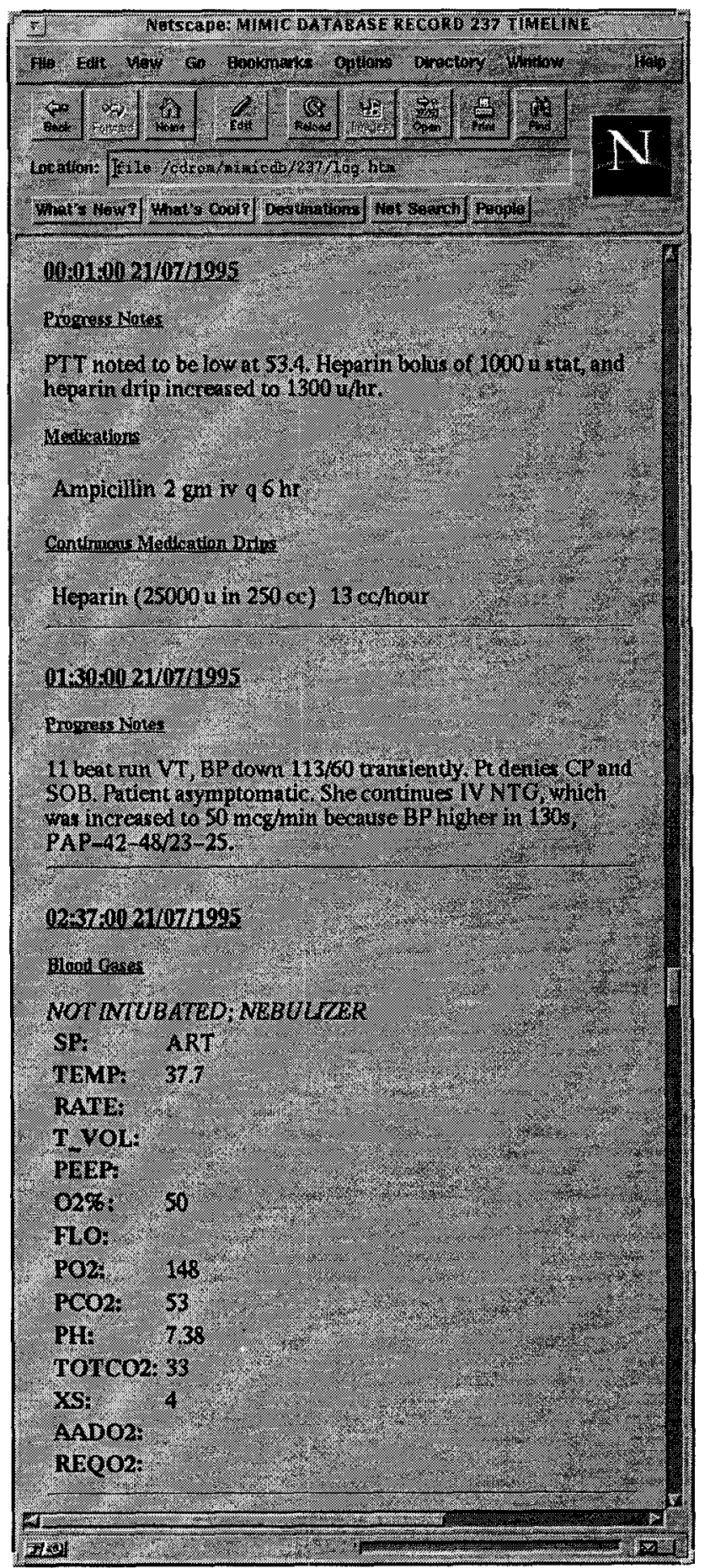

Figure 3: A portion of the HTML time-line file for MIMIC Database record 237. The time-stamp hyperlinks can be selected to examine the recorded signals using WAVE; the other hyperlinks lead to files that contain all data of the specified type (for example, the last link above leads to a table of all blood gas measurements throughout the record). 
responding portion of the recorded physiologic signals and measurements. (This last feature has been implemented by supplying short WAVE script files as the targets of the time-stamp hyperlinks; these files have a distinctive MIME type, so that the browser recognizes them.)

\section{Discussion and Conclusions}

Among the intended applications of the MIMIC Database is what may be termed the virtual patient, by which is meant a simulation of the inputs that would be accessible to a patient monitoring system. Using the time stamps attached to the clinical data, it is possible to reveal the status of the virtual patient as a simulation progresses; thus an algorithm under test can be allowed access to all information known about the patient as of any given time. A limitation of our data collection system, however, is that the time stamps refer to the times at which tests (measurements, specimens, $\mathrm{X}$-ray images) were obtained, and do not indicate when the results of these tests became available (and thus might have influenced the course of treatment). It is of course possible to simulate these delays as well.

Another limitation of any recorded database such as the MIMIC Database or the similarly-motivated IMPROVE Database [3] is that simulations based on recordings cannot incorporate feedback from the monitoring system in the simulation. For example, a future decision support system might request information that is readily available in the ICU but has not been included in the medical record. Even current monitoring systems are designed to influence the care of the patient, by their identification of alarm conditions. Using a recorded simulation, the monitor is restricted to the status of an observer rather than that of a participant in the care of the virtual patient; hence there remain aspects of monitor performance that will still require "real-world" testing. Nevertheless, such realworld testing, apart from its cost, is also of limited value, since it is not strictly reproducible, as a test using a recorded simulation can be.
In order to construct the MIMIC Database, it is necessary for us to create a computer-readable medical record for each patient, based on the information available to us. Doing so is a very expensive, labor-intensive task; certainly, our work would have been very greatly simplified if on-line medical records had been available during the past several years. As observed by Tufte [4],

[Medical records] accumulate bits and pieces of information for administrative, regulatory, financial, legal, and clinical purposes. Largely a device for storage (a write-only memory!), the chart was not designed with the medical treatment of the patient in mind.

We might expect that a well-designed system for realtime on-line medical record entry would incorporate many of the data verification methods and consistency checks that we employed during the transcription process, and that these procedures would result in improvements in the accuracy, completeness, organization, and usability of medical records.

\section{Acknowledgments}

We are grateful for the generous and continuing support of Nihon Kohden America, Inc., and of the Hewlett Packard Foundation.

\section{References}

[1] Moody GB, Mark RG. A database to support development and evaluation of intelligent intensive care monitoring. Computers in Cardiology 1996; 657-660.

[2] Moody GB, Mark RG. The MIT-BIH arrhythmia database on CD-ROM and software for use with it. Computers in Cardiology 1990; 185-188.

[3] IMPROVE project: see http://www.vtt.fi/tte/samba/projects/improve/improve.htm

[4] Tufte ER. Visual Explanations, p. 110. Cheshire, CT (USA): Graphics Press, 1997.

Address for correspondence:

George B. Moody

MIT Room E25-510D

Cambridge, MA 02139 USA

george hs tbme . mit. edu

http://ecg.mit.edu 